\title{
A TYPE OF CONVEXITY IN THE SPACE OF $n$-COMPLEX VARIABLES $\left({ }^{1}\right)$
}

\author{
BY \\ K. DELEEUW
}

Introduction. This paper consists for the main part of a proof of a result in the theory of several complex variables that was obtained as an essential link in a study of the classification of homogeneous Banach algebras on compact abelian groups. (For applications of the result, see [3] and [4].)

Let $X$ be a compact subset of $C^{n}$, the space of $n$-complex variables. $C_{P}(X)$ will denote the set of all points $y$ in $C^{n}$ that satisfy for each polyno$\operatorname{mial} f$

$$
|f(y)| \leqq \operatorname{Sup}_{x \in X}|f(x)|
$$

Equivalently, $C_{P}(X)$ is the intersection of all sets of the form

$$
\{z:|f(z)| \leqq r, f \text { a polynomial }\}
$$

that contain $X$. Thus $C_{P}(X)$ is closed and will be compact since it is contained in any cube $\left\{\left(z_{1}, \cdots, z_{n}\right):\left|z_{i}\right| \leqq K\right\}$ that contains $X .\left(C_{P}(X)\right.$ can be identified in a natural way with the maximal ideal space of the algebra obtained by taking the uniform closure of polynomials on $X$. However we shall not go into this here. See [7].)

It is not hard to see that in the case $n=1, C_{P}(X)$ consists of the union of $X$ and all of the bounded components of the complement of $X$. For $n>1$ no such simple result can be expected. Our main theorem shows that if $X$ is circular in a certain sense, $C_{P}(X)$ is essentially the intersection of all sets of the form

$$
\left\{\left(z_{1}, \cdots, z_{n}\right):\left|z_{1}^{m_{1}} \cdots z_{n}^{m_{n}}\right| \leqq r\right\}
$$

which contain $X$. The second theorem below characterizes the circular sets $X$ that satisfy $C_{P}(X)=X$ as the domains of convergence of power series of a certain type. In [4] we study the representation of functions by power series and Cauchy-type integral formulas on such domains. In all that follows the letters $t$ through $z$ will be used to denote points in $C^{n}$, the space of $n$-complex variables. The letters $a$ through $d$ will denote points in $R^{k}$, Euclidean space of dimension $k$. The $i$ th coordinate of a point will always be denoted by the name of the point with the subscript $i$ attached. Thus $z$ is the point

Presented to the Society, November 26, 1955; received by the editors February 3, 1956.

(1) Research supported by the Office of Naval Research. 
$\left(z_{1}, \cdots, z_{n}\right)$. It will be convenient to define addition and multiplication of points coordinatewise. Thus $z+w$ is the point that has $z_{i}+w_{i}$ as its $i$ th coordinate and $z w$ is the point that has $z_{i} w_{i}$ as its $i$ th coordinate.

1. Identification of $C_{P}(X)$ in the circular case. Let $H$ be a finitely generated abelian group and $\left\{s_{1}, \cdots, s_{n}\right\}$ any set of elements that generate $H$. Let $S$ be the subsemigroup of $H$ generated by the $s_{i}$. That is, $S$ consists of all $s_{1}^{m_{1}} \cdots s_{n}^{m_{n}}$ with the $m_{i}$ non-negative integers not all zero. We shall denote by Hom $(S, C)$ the set of all maps $\phi$ of $S$ into the complex numbers that satisfy

$$
\phi\left(s s^{\prime}\right)=\phi(s) \phi\left(s^{\prime}\right)
$$

and which take the value one on the unit of $H$ if it is in $S$. If the unit of $H$ is not in $S$ we do not exclude the case of $\phi$ being identically zero. by

Hom $(S, C)$ will be considered to be imbedded in $C^{n}$ by the map $\mu$ defined

$$
\mu(\phi)=\left(\phi\left(s_{1}\right), \cdots, \phi\left(s_{n}\right)\right) .
$$

$\mu$ is one-one because the $s_{i}$ generate $S$. We shall denote the image of Hom $(S, C)$ by $V$ and in all that follows consider Hom $(S, C)$ to be identified with this subset of $C^{n}$. $V$ will later be shown to be a subvariety of $C^{n}$. In the simplest case when there are no relations between the $s_{i}$, that is when they are free generators for $H, V$ is all of $C^{n}$.

A subset $W$ of $C^{n}$ is called $V$-circular if $W$ is contained in $V$ and if whenever the point $w$ is in $W$, the point $v$ is in $V$ and $\left|v_{i}\right|=\left|w_{i}\right|$ for all $i$, the point $v$ must be in $W$. In the case that $V$ is all of $C^{n}$ this is the circularity condition that appears in the definition of a Reinhardt domain [1].

For $X$ a compact subset of $V$, denote by $C_{M}(X)$ the intersection of $V$ and all sets of the form

$$
\left\{z:\left|z_{1}^{m_{1}} \cdots z_{n}^{m_{n}}\right| \leqq r, m_{i} \text { non-negative integers }\right\}
$$

that contain $X$. Note that $C_{M}(X)$ is compact and $V$-circular. Our main result is

THEOREM 1. Let $X$ be a compact subset of $C^{n}$ which is $V$-circular. Then $C_{P}(X)=C_{M}(X)$.

This result, even for the special case $V=C^{n}$, does not seem to appear in the literature. For a somewhat related result concerning the characterization of maximal Reinhardt domains see [2, p. 103].

The proof proceeds by a sequence of lemmas. In all that follows we shall assume that $X$ is a subset of $C^{n}$ that satisfies the hypotheses of Theorem 1 .

The first lemma deals with properties of $V$ which will be used often in the constructions that follow. They are immediate consequences of the corresponding properties of $\operatorname{Hom}(S, C)$. 
Lemma 1.1. Let the $z$ and $w$ be points in $V$. Define $x$ by $x_{i}=0$ if $z_{i}=0$ and $x_{i}=1$ otherwise. Define $y$ by $y_{i}=0$ if $z_{i}=0$ and $y_{i}=z_{i}^{-1}$ otherwise. Define $u$ by $u_{i}=\exp \left(t \log \left|z_{i}\right|\right)$ if $z_{i} \neq 0$ and $u_{i}=0$ if $z_{i}=0$, where $t$ is a complex number. Then the points $x, y$ and $u$ are in $V$ and the point zw is in $V$ unless perhaps all of the $z_{i} w_{i}$ are zero.

Proof. The point $x$ is in $V$ because if $\phi$ is in $\operatorname{Hom}(S, C)$, the function $\psi$ defined on $S$ by $\psi(s)=0$ if $\phi(s)=0$ and $\psi(s)=1$ otherwise, is in Hom $(S, C)$. The point $y$ is in $V$ because for $\phi$ in Hom $(S, C)$, the function $\psi$ defined on $S$ by $\psi(s)=0$ if $\phi(s)=0$ and $\psi(s)=(\phi(s))^{-1}$ otherwise, is in Hom $(S, C)$. Similarly the point $u$ is in $V$, because if $\phi$ is in $\operatorname{Hom}(S, C)$, the function $\psi$ defined on $S$ by $\psi(s)=\exp (t \log |\phi(s)|)$ is in $\operatorname{Hom}(S, C)$. The last assertion of the lemma follows because if $\phi_{1}$ and $\phi_{2}$ are in $\operatorname{Hom}(S, C)$, the function $\psi$ defined on $S$ by $\psi(s)=\phi_{1}(s) \phi_{2}(s)$ is in Hom $(S, C)$ except perhaps if it is identically zero.

LemMa 1.2. $V$ is a subvariety of $C^{n}$.

Proof. We shall consider here monomials $X_{1}^{m_{1}} \cdots X_{n}^{m_{n}}$ with the $m_{i}$ nonnegative integers not all zero. Let $M$ be the set of all pairs $(h, j)$ of such monomials which satisfy in $S$

$$
h\left(s_{1}, \cdots, s_{n}\right)=j\left(s_{1}, \cdots, s_{n}\right) .
$$

If the unit of $H$ is in $S$, there is a monomial $g$ of the same type such that $g\left(s_{1}, \cdots, s_{n}\right)$ is equal to the unit. Then $V$ is the set of all points $z$ in $C^{n}$ that satisfy for all $(h, j)$ in $M$

$$
h\left(z_{1}, \cdots, z_{n}\right)=j\left(z_{1}, \cdots, z_{n}\right)
$$

if there is no unit in $S$ and that satisfy these conditions plus the condition $g\left(z_{1}, \cdots, z_{n}\right)=1$ if $S$ has a unit.

Lemma 1.3. $C_{P}(X)$ is contained in $C_{M}(X)$.

Proof. First, $C_{P}(X)$ is contained in $V$. For if the point $z$ is not in $V$, because $V$ is a variety, there is a polynomial $Q$ that satisfies $Q(z) \neq 0$ while vanishing on $V$ and thus $X$. Therefore any $z$ not in $V$ is not in $C_{P}(X)$. By definition $C_{P}(X)$ is the intersection of all sets of the form

$$
\{z:|f(z)| \leqq r, f \text { a polynomial }\}
$$

that contain $X$. But $C_{M}(X)$ is the intersection of a smaller class of sets and $V$. Since $V$ has been shown to contain $C_{P}(X)$, it follows that $C_{P}(X)$ is a subset of $C_{M}(X)$.

Lemma 1.4. To prove Theorem 1 , it suffices to prove that $C_{M}\left(C_{P}(X)\right)=C_{P}(X)$.

Proof. Since $X \subset C_{P}(X)$, it follows that $C_{M}(X) \subset C_{M}\left(C_{P}(X)\right)$. Thus if it is true that $C_{M}\left(C_{P}(X)\right)=C_{P}(X)$, we have $C_{M}(X) \subset C_{P}(X)$. This in conjunction 
with Lemma 1.3 shows that $C_{M}(X)=C_{P}(X)$, which is the conclusion of Theorem 1.

The next lemma is a criterion that is sufficient to insure that a subset $Y$ of $C^{n}$ satisfy $C_{M}(Y)=Y$. This criterion will later be shown to hold for $Y=C_{P}(X)$ and Lemma 1.4 will complete the proof of Theorem 1. This criterion will also be used in the next section in the discussion of power series. First some definitions are needed. Let $k$ be any integer satisfying $1 \leqq k \leqq n$. Let $K$ be any $k$-tuple of distinct positive integers $\left(r_{1}, \cdots, r_{k}\right)$, where each of the $r_{i} \leqq n$. Let $C^{K}$ be the subset of $C^{n}$ that consists of those $z$ having $z_{m} \neq 0$ if and only if $m$ occurs in $K$. Define the map $L_{K}: C^{K} \rightarrow R^{k}$ by

$$
L_{K}(z)=\left(\log \left|z_{r_{1}}\right|, \cdots, \log \left|z_{r_{n}}\right|\right) .
$$

Lemma 1.5. Let $Y$ be a compact subset of $C^{n}$ that is $V$-circular. Assume that $Y$ satisfies the two hypotheses: 1 . If $z$ is in $Y$, w is in $V$ and $\left|w_{i}\right| \leqq\left|z_{i}\right|$, then the point w must be in $Y .2$. For each $K$, the sets $L_{K}\left(C^{K} \cap Y\right)$ are either empty or convex. Then $C_{M}(Y)=Y$.

Proof. Let $x$ be any point in $V$ that is not in $Y$. To prove the lemma, it is sufficient to find a monomial $f$ that satisfies

$$
|f(x)|>\operatorname{Sup}_{\nu \in Y}|f(y)| \text {. }
$$

Let $K$ be the set of all $m$ with $x_{m} \neq 0$. We shall assume that the coordinates have been rearranged so that $K$ is $(1,2, \cdots, k)$. With $L_{K}$ as defined above, the set $L_{K}\left(C^{K} \cap V\right)$ will be denoted by $V_{K}$ and the set $L_{K}\left(C^{K} \cap Y\right)$ by $Y_{K}$. It will first be shown that $V_{K}$ is a linear subspace of $R^{k}$. Take any point $w$ in $C^{K} \cap V$. Let $r$ be any real number and define $u$ in $C^{K}$ by $u_{i}=\left|w_{i}\right| r$ if $w_{i} \neq 0$ and $u_{i}=0$ if $w_{i}=0$. Because of Lemma 1.1, $u$ is in $C^{K} \cap V$, and $L_{K}(u)=r L_{K}(w)$. This together with the fact that $L_{K}(x y)=L_{K}(x)+L_{K}(y)$ for any two points $x$ and $y$ in $C^{K} \cap V$ show that $V_{K}$ is a linear subspace of $R^{k}$. It will be shown next that any point $a$ having $a_{i} \leqq \log \left|x_{i}\right|$ and lying in the subspace $V_{K}$ must be in $Y_{K}$. Let $a=L_{K}(v)$ for some $v$ in $C^{K} \cap V$. Then $\exp \left(a_{i}\right)=\left|v_{i}\right|$ for $i \leqq k$ and because of Lemma 1.1 the point

$$
u=\left(e^{a_{1}}, \cdots, e^{a_{k}}, 0, \cdots, 0\right)
$$

will be in $C^{K} \cap V$. Since $\left|e^{a_{i}}\right| \leqq\left|x_{i}\right|$ and the point $\left(\left|x_{1}\right|, \cdots,\left|x_{n}\right|\right)$ is in $Y$, hypothesis 1 shows that $u$ will be in $C^{K} \cap Y$. Thus $a=L_{K}(u)$ is in $Y_{K}$ and we have shown that any point $a$ in $V_{K}$ with $a_{i} \leqq \log \left|x_{i}\right|$ must be in $Y_{K}$. At this point the convexity of $Y_{K}$ given by hypothesis 2 will be used. The point $L_{K}(x)$ is not in $Y_{K}$, because if it were, by the circularity of $Y$, we would have $x$ in $Y . Y_{K}$ is closed and because it is convex there is a hyperplane in $V_{K}$ that separates the point $L_{K}(x)$ from the set $Y_{K}$. That is, there is a linear functional $\gamma: V_{K} \rightarrow R$ that satisfies $\gamma\left(L_{K}(x)\right)>C$ and $\gamma\left(Y_{K}\right)<C$, for some constant $C$. If $a$ is any point in $V_{K}$ with all $a_{i}<0, \gamma(a)$ must be nonpositive. For if $\lambda$ is suffi- 
ciently large, $\lambda a_{i}<\log \left|x_{i}\right|$ so that the point $\lambda a$, will be in $Y_{K}$. Since $\gamma(\lambda a)$ $=\lambda(\gamma(a)), \gamma(a)$ must be nonpositive since $\gamma$ is bounded from above on $Y_{K}$. It is known $\left(^{2}\right)$ that such a linear functional can be extended to a positive linear functional $\bar{\gamma}$ on all of $R^{k}$. That is, $\bar{\gamma}(c)=\gamma(c)$ for $c$ in $V_{K}$ and $\bar{\gamma}(c) \geqq 0$ if $c_{i} \geqq 0$ for all $i$. There are non-negative constants $d_{i}$ so that $\bar{\gamma}(a)=d_{1} a_{1}+\cdots+d_{k} a_{k}$. The composite mapping $\bar{\gamma} L_{K}$ on $C^{K}$ is

$$
\bar{\gamma} L_{K}(z)=\sum_{i=1}^{k} d_{i} \log \left|z_{i}\right| .
$$

It has been shown that this function is greater on the point $x$ than its sup on $C^{K} \cap Y$. The function $g: C^{n} \rightarrow R$ defined by

$$
g(z)=\prod_{i=1}^{k}\left|z_{i}\right|^{d_{i}}
$$

thus also satisfies

$$
\operatorname{Sup}_{y \in C^{K} \cap Y}|g(y)|<|g(x)| \text {. }
$$

Since this property remains valid if all of the $d_{i}$ are increased slightly, we may assume that all of the $d_{i}$ are strictly positive. We shall use the fact that $|g|$ depends on all $z_{i}$ for $i \leqq k$ and is independent of the $z_{i}$ with $i>k$ to show that its sup on $Y$ is no greater than its sup on $C^{K} \cap Y$. Take any point $y$ in $Y$. Assume first that none of the $y_{i}$ for $i \leqq k$ are zero. Then because of Lemma 1.1 the point $y^{\prime}=\left(y_{1}, \cdots, y_{k}, 0, \cdots, 0\right)$ is in $V$. Hypothesis 1 insures that the point $y^{\prime}$ is in $Y$ and thus in $C^{K} \cap Y$. Since $g\left(y^{\prime}\right)=g(y)$, no point $y$ in $Y$ with $y_{i} \neq 0$ for $i \leqq k$ can increase the value of $|g|$ over its sup on $C^{K} \cap Y$. If on the other hand the point $y$ has $y_{i}=0$ for some $i \leqq k, g(y)=0$. Thus

$$
\operatorname{Sup}_{y \in Y}|g(y)|=\operatorname{Sup}_{y \in C^{R} \cap Y}|g(y)|<|g(x)| .
$$

By taking positive rational approximations $e_{i}$ to the $d_{i}$ one can find a function $g^{\prime}: C^{n} \rightarrow R$ defined by

$$
g^{\prime}(z)=\prod_{i=1}^{k}\left|z_{i}\right|^{e_{i}}
$$

that also satisfies

$$
\operatorname{Sup}_{y \in Y}\left|g^{\prime}(y)\right|<\left|g^{\prime}(x)\right| \text {. }
$$

Let $e_{i}=f_{i} / u$ with the $f_{i}$ and $u$ non-negative integers. Then the monomial $f\left(z_{1}, \cdots, z_{n}\right)=z_{1}^{f_{1}} \cdots z_{k}^{f_{k}}$ has the property that is wanted:

(2) A proof of this is given in [5]. 


$$
|f(x)|>\operatorname{Sup}_{\nu \in Y}|f(y)| .
$$

This concludes the proof of Lemma 1.5.

It can be shown that a converse to Lemma 1.5 is valid, namely that if $Y$ is a subset of $C^{n}$ that satisfies $C_{M}(Y)=Y$, then $Y$ must satisfy hypotheses 1 and 2 of Lemma 1.5 .

Before we shall be in a position to prove that hypothesis 1 of Lemma 1.5 is valid for $Y=C_{P}(X)$, two preliminary results are needed.

Lemma 1.6. Let $T$ be the set of all $z$ in $V$ that satisfy $\left|z_{i}\right|=1$ for all $i$. $A$ subset $Y$ of $V$ is $V$-circular if and only if it satisfies the following criterion: If $w$ is in $Y$ and $t$ is in $T$, the point tw is in $Y$.

Proof. The criterion is satisfied for a $V$-circular set, for $t w$ must be on $V$ by Lemma 1.1 and $\left|t_{i} w_{i}\right|=\left|w_{i}\right|$ for all $i$. For the converse, assume that a subset $Y$ of $V$ satisfies the criterion. Let $z$ be in $V$ and $w$ be in $Y$ with $\left|z_{i}\right|=\left|w_{i}\right|$. We shall have shown $Y$ to be $V$-circular if a point $t$ on $T$ can be found that satisfies $z_{i}=t_{i} w_{i}$ for all $i$. Let $K$ be the set of all $i$ with $w_{i} \neq 0$. Let $H_{K}$ be the subgroup of the group $H$ defined previously that is generated by the $s_{i}$ for $i$ in $K$. There is a character $\phi_{K}: H_{K} \rightarrow C$ determined by $\phi_{K}\left(s_{i}\right)=z_{i} / w_{i}$. This can be extended (see [6]) to a character $\phi$ of $H$. If $\phi$ is restricted to $S \subset H$ it can be considered to be an element in $\operatorname{Hom}(S, C)$. Let $t_{i}=\phi\left(s_{i}\right)$. Then $t$ is in $T$ and satisfies $z_{i}=t_{i} w_{i}$.

This proof shows that $T$ acts as a group of homeomorphisms of $Y$ if $Y$ is $V$-circular. It can also be shown that under the identification of Hom $(S, C)$ with $V$, a subgroup of $\operatorname{Hom}(S, C)$ that is canonically isomorphic to $H^{*}$, the dual of $H$, is identified with $T$. Thus $H^{*}$ acts on all $V$-circular sets.

Lemma 1.7. $C_{P}(X)$ is V-circular.

Proof. If we are given any points $w$ in $C_{P}(X)$ and $t$ in $T$, Lemma 1.6 shows that to demonstrate that $C_{P}(X)$ is $V$-circular it is sufficient to show that the point $t w$ must be in $C_{P}(X)$. Since $w$ is in $C_{P}(X)$, if $f$ is any polynomial

$$
|f(w)| \leqq \operatorname{Sup}_{z \in X}|f(z)| .
$$

Let $g$ be any polynomial. Define a function $f$ in $C^{n}$ by $f\left(z_{1}, \cdots, z_{n}\right)$ $=g\left(t_{1} z_{1}, \cdots, t_{n} z_{n}\right)$. Then $f$ is a polynomial and because of (1)

$$
|g(t w)| \leqq \operatorname{Sup}_{z \in X}|g(t z)| \text {. }
$$

But because $X$ is $V$-circular, Lemma 1.6 shows that the points $t z$ cover $X$ as $z$ runs over $X$. Thus we have

$$
|g(t w)| \leqq \operatorname{Sup}_{z \in X}|g(z)|
$$


so that $t w$ is in $C_{P}(X)$, proving that $C_{P}(X)$ is $V$-circular.

We are now in a position to prove that hypothesis 1 of Lemma 1.5 is valid in the case $Y=C_{P}(X)$.

LEMMA 1.8. Let $z$ be any point in $C_{P}(X)$. Let $w$ be a point in $V$ that satisfies $\left|w_{i}\right| \leqq\left|z_{i}\right|$ for all $i$. Then $w$ must be in $C_{P}(X)$.

Proof. We shall show first that it is sufficient to prove this in the case that the $z_{i}$ and $w_{i}$ are non-negative real numbers. By the circularity of $C_{P}(X)$ proved in Lemma 1.7, if $z$ is in $C_{P}(X)$, the point $\left(\left|z_{1}\right|, \cdots,\left|z_{n}\right|\right)$ is in $C_{P}(X)$. If $w$ is in $V$, Lemma 1.1 shows that $\left(\left|w_{1}\right|, \cdots,\left|w_{n}\right|\right)$ is also in $V$. Thus if we knew Lemma 1.8 to be valid in the case that all of the components of $w$ and $z$ were non-negative real numbers, we could conclude that $\left(\left|w_{1}\right|, \cdots,\left|w_{n}\right|\right)$ would be in $C_{P}(X)$. By the circularity of $C_{P}(X)$, w would be in $C_{P}(X)$. We may therefore assume that $w_{i} \geqq 0$ and $z_{i} \geqq 0$. The result wanted will be shown to follow from the two extreme cases that will be treated first. Case $I, w_{i}$ is equal to either $z_{i}$ or zero; Case II, $w_{i}$ is zero if and only if $z_{i}$ is zero. These cases will be treated simultaneously. Because of Lemma 1.1 there is a $\phi$ in Hom $(S, C)$ that satisfies $\phi\left(s_{i}\right)=0$ if $w_{i}=0$ and $\phi\left(s_{i}\right)=1$ if $w_{i} \neq 0$. Lemma 3.2, which will be proved later, shows that there is a homomorphism $g$ of $S$ into the additive semigroup of non-negative reals that satisfies $g\left(s_{i}\right)=0$ if $w_{i} \neq 0$ and $g\left(s_{i}\right)>0$ if $w_{i}=0$. In case I let $\lambda_{i}=-g\left(s_{i}\right)$. In case II let $\lambda_{i}=\log \left(w_{i} / z_{i}\right)$ if $z_{i}$ is nonzero and $\lambda_{i}=0$ otherwise. In both cases the association $s_{i} \rightarrow \lambda_{i}$ induces a homomorphism of $S$ into the additive reals, so that for every complex number $u$ there is a $\psi$ in $\operatorname{Hom}(S, C)$ with

$$
\psi\left(s_{i}\right)=e^{\lambda_{i u}} .
$$

This observation and Lemma 1.1 show that in both cases, for every complex number $u$, the point

$$
\left(z_{1} e^{\lambda_{1} u}, \cdots, z_{n} e^{\lambda_{n} u}\right)
$$

will be in $V$. Since $z$ is in $C_{P}(X)$ which is $V$-circular, if $\operatorname{Re}(u)=0$, this point will actually be in $C_{P}(X)$. For any polynomial $f$ in $C^{n}$, define $f^{*}$ by

$$
f^{*}(u)=f\left(z_{1} e^{\lambda_{1} u}, \cdots, z_{n} e^{\lambda_{n} u}\right) .
$$

$f^{*}$ is an exponential polynomial which is bounded in the half-plane $\operatorname{Re}(u) \geqq 0$ since the $\lambda_{i}$ are non-positive. Thus by the maximum modulus principle, if $\operatorname{Re}(u) \geqq 0$,

$$
\left|f^{*}(u)\right| \leqq \operatorname{Sup}_{\operatorname{Re}(\boldsymbol{v})=0}\left|f^{*}(v)\right|
$$

In case I,

$$
f(w)=\lim _{\sigma \rightarrow+\infty} f^{*}(\sigma+i 0)
$$


so that

$$
|f(w)| \leqq \operatorname{Sup}_{\operatorname{Re}(v)=0}\left|f^{*}(v)\right| \leqq \operatorname{Sup}_{x \in C P(X)}|f(x)|,
$$

the second inequality following from the previous observation that the point (2) is in $C_{P}(X)$ if $\operatorname{Re}(u)=0$. Since this inequality holds for all polynomials $f$, $w$ in case $\mathrm{I}$ is in $C_{P}\left(C_{P}(X)\right)$ which is $C_{P}(X)$. In case II

$$
f(w)=f^{*}(1)
$$

so that again one has the inequalities (3) and $w$ is in $C_{P}(X)$. The general case now follows from I and II. Define the point $v$ by $v_{i}=z_{i}$ if $w_{i} \neq 0$ and $v_{i}=0$ if $w_{i}=0$. Lemma 1.1 shows that $v$ is in $V$. By case $I$ applied to $z$ and $v$, the point $v$ must be in $C_{P}(X)$. Now case II applied to $v$ and $w$ shows that $w$ must be in $C_{P}(X)$. Thus Lemma 1.8 is proved.

It will now be shown that the second hypothesis of Lemma 1.5 is valid for $C_{P}(X)$.

Lemma 1.9. $L_{K}\left(C^{K} \cap C_{P}(X)\right)$ is either empty or convex.

Proof. Let $w$ and $z$ be any two points in $C^{K} \cap C_{P}(X)$. It suffices to show that the point

$$
t L_{K}(w)+(1-t) L_{K}(z)
$$

is in $L_{K}\left(C^{K} \cap C_{P}(X)\right)$ for $0 \leqq t \leqq 1$. We may assume that $w_{i} \geqq 0$ and $z_{i} \geqq 0$ since $C_{P}(X)$ is $V$-circular and

$$
L_{K}\left(z_{1}, \cdots, z_{n}\right)=L_{K}\left(\left|z_{1}\right|, \cdots,\left|z_{n}\right|\right) .
$$

Let $\lambda_{i}=\log \left(z_{i} / w_{i}\right)$ if $i$ is in $K$ (that is, if $z_{i}$ and $w_{i}$ are nonzero) and $\lambda_{i}=0$ otherwise. Several applications of Lemma 1.1 show that for every complex $t$, the point

$$
p_{t}=\left(w_{1} e^{\lambda_{1} t}, \cdots, w_{n} e^{\lambda_{n} t}\right)
$$

is in $V$. Since $w$ is in $C_{P}(X)$ which is $V$-circular, if $\operatorname{Re}(t)=0, p_{t}$ is actually in $C_{P}(X)$. If $t=1, p_{t}$ is $z$ which is in $C_{P}(X)$. Thus if $\operatorname{Re}(t)=1, p_{t}$ is also in $C_{P}(X)$. For any polynomial $f$ in $C^{n}$ define $f^{*}$ by

$$
f^{*}(t)=f\left(p_{t}\right) .
$$

$f^{*}$ is an exponential polynomial with real exponents and so is bounded in the strip $0 \leqq \operatorname{Re}(t) \leqq 1$. Thus, because of the maximum modulus principle, if $0 \leqq t \leqq 1$,

$$
\left|f\left(p_{t}\right)\right|=\left|f^{*}(t)\right| \leqq \operatorname{Sup}_{\operatorname{Re}(v)=0 \text { or } 1}\left|f^{*}(v)\right| \leqq \operatorname{Sup}_{v \in C_{P}(x)}|f(y)|
$$

with the last inequality following from the previous observation that if $\operatorname{Re}(v)$ is 0 or 1 that $p_{t}$ is in $C_{P}(X)$. Since this is valid for any polynomial $f$, 
$p_{t}$ is in $C_{P}\left(C_{P}(X)\right)$ which is $C_{P}(X)$. Thus

$$
L_{K}\left(p_{t}\right)=t L_{K}(w)+(1-t) L_{K}(z)
$$

is in $L_{K}\left(C^{K} \cap C_{P}(X)\right)$ and Lemma 1.9 is proved.

It is now possible to complete the proof of Theorem 1. Because of Lemma 1.4 it suffices to prove that $C_{M}\left(C_{P}(X)\right)=C_{P}(X)$. Lemma 1.5 gives a criterion that is sufficient for $C_{M}(Y)=Y$ and Lemmas 1.7, 1.8 and 1.9 show that the criterion is applicable to $Y=C_{P}(X)$.

2. Power series in Hom $(S, C)$. We shall be concerned here with formal series

$$
\sum_{s \in S} \alpha_{8}^{8}
$$

where the $\alpha_{8}$ are complex numbers. The convergence set of such a series is the set of all $\phi$ in $\operatorname{Hom}(S, C)$ that satisfy

$$
\sum_{s \in S}\left|\alpha_{s} \phi(s)\right|<\infty \text {. }
$$

If $\phi$ is in the convergence set, the series $\sum \alpha_{8} \phi(s)$ converges to some value $f(\phi)$, independently of the order of summation, so that the series can be considered to define the function $f$ on its convergence set.

Let $s_{1}, \cdots, s_{n}$ be the generators of $S$ that were discussed earlier. To each $s$ in $S$ associate some one $n$-tuple of non-negative integers $\left(m_{1}, \cdots, m_{n}\right)$ which has the property that $s=s_{1}^{m_{1}} \ldots s_{n}^{m_{n}}$. Define $\alpha\left(m_{1}, \cdots, m_{n}\right)$ to be $\alpha_{n}$ in this case. Then the subset of $V$ that corresponds to the convergence set of (1), under the identification of $\operatorname{Hom}(S, C)$ with $V$ given by

$$
\mu(\phi)=\left(\phi\left(s_{1}\right), \cdots, \phi\left(s_{n}\right)\right),
$$

is precisely the set of points in $C^{n}$ which lie on $V$ and at which the series

$$
\sum \alpha\left(m_{1}, \cdots, m_{n}\right) z_{1}^{m_{1}} \cdots z_{n}^{m_{n}}
$$

converges absolutely. The following characterization of compact subsets of $V$ that are identified with convergence sets is an immediate consequence of known results in the case that $V$ is all of $C^{n}$ (see [1]). Using Lemma 1.5 it is not much harder in the general case.

THEOREM 2. Let $Y$ be a compact subset of $V$ that is identified by the mapping $\mu$ with the convergence set of a series $\sum \alpha_{s}$ s. Then $C_{M}(Y)=Y$. Conversely let $Y$ be a compact subset of $V$ that satisfies $C_{M}(Y)=Y$. Then there is a series $\sum \alpha_{s}$ s which has the property that $Y$ is identified with its convergence set by $\mu$.

Proof. Lemma 1.5 will be used to establish the first implication. It is clear that $Y$ is $V$-circular and that hypothesis 1 of Lemma 1.5 is satisfied. The convexity in hypothesis 2 is proved exactly as in the classical case (see 
for example [8]) and $C_{M}(Y)=Y$ follows. Now let $Y$ be any compact subset of $V$ that satisfies $C_{M}(Y)=Y$. The second implication will be established by constructing a series that has $\mu^{-1}(Y)$ its convergence set. The fact that $C_{M}(Y)=Y$ implies that $\mu^{-1}(Y)$ is the intersection of all sets of the form

$$
\{\phi:|\phi(s)| \leqq r, s \text { fixed in } S\}
$$

that contain it. Let $p(s)=\operatorname{Sup}|\phi(s)|$ with $\phi$ running over $\mu^{-1}(Y)$. It is finite since $Y$ is compact. Then

$$
\mu^{-1}(Y)=\bigcap_{s \in S}\{\phi:|\phi(s)| \leqq p(s)\} .
$$

Let $s_{1}, s_{2}, \cdots, s_{r}, \cdots$ be a particular enumeration of $S$. For fixed $r$ the convergence set of the series

$$
\sum_{n=1}^{\infty} \frac{1}{n^{2} p\left(s_{r}\right)^{n}} s_{r}^{n}
$$

is $\left\{\phi:\left|\phi\left(s_{r}\right)\right| \leqq p\left(s_{r}\right)\right\}$. Thus the series

$$
\sum_{r=1}^{\infty} \frac{1}{2^{r}}\left(\sum_{n=1}^{\infty} \frac{1}{n^{2} p\left(s_{r}\right)^{n}} s_{r}^{n}\right)
$$

which is of type (1) when rearranged, has $\mu^{-1}(Y)$ as its convergence set. The proof of Theorem 2 is complete.

3. Convex cones and homomorphisms of $S$. This section is devoted to the proof of a result, which concerns the existence of homomorphisms of $S$ in to the additive semigroup $R^{+}$of non-negative reals and which was used in the proof of Lemma 1.8. Let $g: S \rightarrow R^{+}$be such a homomorphism. Define the function $\phi$ on $S$ by $\phi(s)=1$ if $g(s)=0$ and $\phi(s)=0$ if $g(s)>0$. Then $\phi$ is an element of Hom $(S, C)$ that takes on only the two values 0 and 1 . The result that we shall prove is that every such $\phi$ arises in the manner indicated from a homomorphism $g: S \rightarrow R^{+}$.

Before proving this, it will be necessary to establish an analogous result for finitely generated subsemigroups in real vector spaces. Let $U$ be a finite dimensional vector space over the reals. Let $p_{1}, \cdots, p_{n}$ be a finite set of points in $U$, and $P$ be the set of all points in $U$ having some representation of the form $\sum r_{i} p_{i}$ with the $r_{i} \geqq 0$. $P$ is to be considered as a semigroup under addition. Let $\theta: P \rightarrow(0,1)$ be a homomorphism of $P$ into the semigroup consisting of the two numbers 0 and 1 under multiplication. We shall assume that $\theta(0, \cdots, 0)=1$. Denote $\theta^{-1}(0)$ by $P_{0}$ and $\theta^{-1}(1)$ by $P_{1}$. The geometrical content of the next lemma is that there is a hyperplane in $U$ that contains $P_{1}$ and which has $P_{0}$ properly on one side.

LeмmA 3.1. There exists a linear functional $\gamma: U \rightarrow R$ that satisfies $\gamma(u)=0$ if $u$ is in $P_{1}$ and $\gamma(u)>0$ if $u$ is in $P_{0}$. 
Proof. Let $W$ be the subspace of $U$ that is generated by $P_{1}$. Then $W$ consists precisely of all $u_{1}-u_{2}$ with $u_{1}$ and $u_{2}$ in $P_{1}$. We shall show that $W$ and $P_{0}$ have no elements in common. Assume to the contrary that there is a $u_{3}$ in $P_{0}$ that satisfies $u_{3}=u_{1}-u_{2}$ with $u_{1}$ and $u_{2}$ in $P_{1}$. Then $u_{3}+u_{2}=u_{1}$. But $\theta\left(u_{3}+u_{2}\right)=\theta\left(u_{3}\right) \theta\left(u_{2}\right)=0$ while $\theta\left(u_{1}\right)=1$ so that no such $u_{3}$ exists. Thus $W \cap P_{0}$ is void. Consider the natural projection $\pi: U \rightarrow U / W$. Let $P^{\prime}$ be the subset of $U / W$ that consists of all

$$
\sum_{p_{i} \in P_{0}} r_{i} \pi\left(p_{i}\right)
$$

with $\sum r_{i}=1, r_{i} \geqq 0$. The origin is not in $P^{\prime}$. For if some $\sum r_{i} \pi\left(p_{i}\right)$ in $P^{\prime}$ were zero, then $\sum r_{i} p_{i}$ would be in $W$ and the intersection of $W$ and $P_{0}$ would be nonvoid. $P^{\prime}$ is a compact convex subset that does not contain the origin and thus can be separated from it by a hyperplane. Equivalently, there is a linear functional $\delta$ to the reals that is positive on $P^{\prime}$. Then $\delta \pi\left(p_{i}\right)>0$ for all the $p_{i}$ in $P_{0}$. The linear functional $\gamma$ that is needed can be taken to be

$$
\gamma=\delta \pi: U \rightarrow R \text {. }
$$

If $u$ is in $P_{0}$ there is some positive multiple $\lambda u$ which is such that $\pi(\lambda u)$ is in $P^{\prime}$ so that $\gamma(u)>0$. If $u$ is in $P_{1}$, then $\gamma(u)=0$ because $\pi(u)=0$. Thus $\gamma$ satisfies the needed conditions.

We can now prove the result used in Lemma 1.8. Let $\phi$ be an element in $\operatorname{Hom}(S, C)$ that takes only the values 0 and 1 . Denote $\phi^{-1}(0)$ by $S_{0}$ and $\phi^{-1}(1)$ by $S_{1}$. Let $R^{+}$be the semigroup of non-negative reals under addition.

LеммA 3.2. There is a homomorphism $g: S \rightarrow R^{+}$that satisfies $g(s)=0$ if $s$ is in $S_{1}$ and $g(s)>0$ if $s$ is in $S_{0}$.

Proof. Recall that $S$ is a subsemigroup of a finitely generated abelian group $H$. Let $F$ be the subgroup of $H$ consisting of all elements of finite order. Denote by $S^{\prime}$ the image of $S$ under the canonical map $\pi: H \rightarrow H / F$. The function $\phi^{\prime}$ defined on $S^{\prime}$ by $\phi^{\prime}(\pi(s))=\phi(s)$ is well defined and a homomorphism. $H / F$ is a finitely generated free abelian group. Let $t_{1}, \cdots, t_{k}$ be a particular set of generators. Let $U$ be a $k$-dimensional vector space over the reals and $u_{1}, \cdots, u_{p}$ a particular basis. There is a unique group homomorphism $\alpha: H / F \rightarrow U$ that is determined by $\alpha\left(t_{j}\right)=u_{j}$. Denote the images under the composite map $\alpha \pi: H \rightarrow U$ of the elements $s_{1}, \cdots, s_{n}$ in $S$ by $p_{1}, \cdots, p_{n}$. Let $P$ be, as in the preceding lemma, the set of all $\sum r_{i} p_{i}$ with $r_{i} \geqq 0$. The homomorphism $\phi^{\prime}$ on $S^{\prime}$ determines in a natural manner a homomorphism $\theta$ of $P$ as follows: Define $\theta$ on $\alpha\left(S^{\prime}\right)$ by $\theta\left(\alpha\left(s^{\prime}\right)\right)=\phi^{\prime}\left(s^{\prime}\right)$. For every $p$ in $P$ except perhaps $p=(0, \cdots, 0)$, there is a positive integer $m$ such that $m p$ is in $\alpha\left(S^{\prime}\right)$. Define $\theta(p)=\theta(m p)$ and $\theta(0, \cdots, 0)=1$. Then $\theta$ is well defined and a homomorphism. If $s$ is in $S$, then $\theta(\alpha \pi(s))=\phi(s)$ and $\theta$ is the only homomorphism of $P$ into the semigroup consisting of 0 and 1 that has this property. We shall 
now use the preceding lemma on the map $\theta$. There is a linear functional $\gamma: U \rightarrow R$ that satisfies, for all $p$ in $P, \gamma(p)=0$ if $\theta(p)=1$ and $\gamma(p)>0$ if $\theta(p)=0$. Thus the composite map

$$
\gamma \alpha \pi: S \rightarrow R
$$

satisfies all the conditions demanded of the map $g$ that is sought.

With a slight modification of the above argument a $g$ can be found that takes only integral values.

\section{BIBLIOGRAPHY}

1. H. Behnke and P. Thullen, Theorie der Functionen mehrer komplexer Veranderlichen, Berlin, 1934.

2. H. Cartan, Les fonctions de deux variables complexes et le problème de la representation analytique, Journal de Mathématique vol. 96 (1931) pp. 1-114.

3. K. deLeeuw, On homogeneous Banach algebras, to appear.

4. - Functions on circular subsets of the space of $n$ complex variables, to appear.

5. H. Mirkil, A new characterization of polyhedral cones; to appear in Canadian Journal of Mathematics.

6. L. Pontrjagin, Topological groups, Princeton, 1939.

7. G. Silov, On decomposition of a commutative normed ring in a direct sum of ideals, Amer. Math. Soc. Translations, Series 2, vol. 1, pp. 37-48.

8. H. Tietze, Über den Bereich absoluten Konvergenz von Potenzreihen mehrer Veranderlichen, Math. Ann. vol. 99 (1928).

UNIVERSITY OF WisCONSIN,

Madison, Wis. 